\title{
Multivariate analysis of a genome-wide association study in dairy cattle
}

\author{
S. Bolormaa, ${ }^{* 1}$ J. E. Pryce, ${ }^{*}$ B. J. Hayes,${ }^{*}$ and M. E. Goddard ${ }^{*} \dagger$ \\ ${ }^{*}$ Biosciences Research Division, Department of Primary Industries Victoria, 1 Park Drive, Bundoora 3083, Australia \\ †School of Land and Environment, University of Melbourne, Parkville 3010, Australia
}

\section{ABSTRACT}

Multiple-trait genome-wide association study (GWAS) analyses were compared with single-trait GWAS for power to discover and subsequently validate genetic markers (single nucleotide polymorphisms; SNP) associated with dairy traits. The SNP associations were discovered in 1 Holstein population and validated in both a Holstein population consisting of bulls younger than those in the discovery population and a Jersey population. The multivariate methods used were a principal component analysis and a series of bivariate analyses. The statistical power of detecting associations using multiple-trait GWAS was as good as or better than that of the best single-trait GWAS. Additional SNP associations were found with the multivariate methods that had not been discovered in the singletrait analyses; this was achieved without an increase in the false discovery rate. From the multivariate analysis, 4 common pleiotropic patterns were identified among the putative quantitative trait loci (QTL) affecting the Australian selection index. These patterns could be interpreted as a primary effect of the putative QTL on 1 or more milk components and secondary effects on other components. The multivariate analysis did not appear to increase the precision with which putative QTL were mapped.

Key words: multivariate genome-wide association study, principal component, pleiotropy

\section{INTRODUCTION}

The availability of dense SNP assays for cattle (e.g., Matukumalli et al., 2009) has made it possible to map genes affecting a quantitative trait throughout the genome using linkage disequilibrium between the marker SNP and the QTL. For instance, Cole et al. (2009) and Pryce et al. (2010a) both describe a genome-wide association study (GWAS) for production and fertility traits in Holstein cattle. Multiple-trait analysis of

Received December 7, 2009.

Accepted April 8, 2010.

${ }^{1}$ Corresponding author: bolormaa.sunduimijid@dpi.vic.gov.au linkage experiments has been reported to increase the power to detect QTL (Knott and Haley, 2000; Korol et al., 2001). This paper investigates whether additional power can be extracted from a GWAS by analyzing traits together rather than one at a time.

When correlated traits are analyzed, the sampling errors tend to be correlated and this makes the interpretation of the results difficult. To overcome this problem, methods that generate uncorrelated traits are useful and have been used in this study. When the effects of SNP on a trait are tested one at a time, which is the typical approach for a GWAS, some account needs to be taken of the multiple testing that arises from performing many significance tests. Unless a very stringent significance threshold is used, a considerable number of false positives are expected, given the large number of SNP that are being tested. However, if the number of significant SNP is greater than the number expected by chance, some of the significant SNP must be true positive findings. This logic has led to the use of a false discovery rate (FDR) rather than a conventional significance test (Benjamini and Hochberg, 1995; Storey, 2002; Osborne, 2006). For instance, an FDR of $10 \%$ implies that $90 \%$ of the significant SNP are true positive findings. The advantage of using an FDR approach is that the same test can be used whether 1 or many traits are tested. That is, the FDR does not need to be adjusted because of the multiple testing caused by testing many traits.

False discovery rate calculations rely on the assumptions of the statistical method used to calculate traditional significance values. A more robust method is to validate the significant associations between traits and SNP in an independent data set. Therefore, a population of Holstein bulls was used to discover associations and 2 independent populations ( 1 of Holstein bulls and 1 of Jersey bulls) were used to validate these associations. Within one breed, substantial linkage disequilibrium $(\mathbf{L D})$ can persist for hundreds of kilobases or even megabases such that SNP that are a substantial physical distance from the QTL can still be associated with it. However, across breeds such as Holstein and Jersey, LD extends only tens of kilobases (de Roos et al., 2008; The Bovine Hap Map Consortium, 2009). This means that 
SNP associations discovered in Holsteins and validated in Jerseys are likely to be very close to the QTL. This across-breed mapping strategy has been used in other species, such as dogs (e.g., Karlsson et al., 2007).

The precision with which a QTL can be positioned on the genome in a GWAS is limited by 2 sources of error. First, the LD between marker SNP and the QTL is highly variable and therefore the nearest SNP is not necessarily the one in greatest LD with the QTL. Second, the LD with the QTL is not observed directly but only via the effect of the QTL on a phenotypic trait. Because the QTL typically explains only a small amount of the variance of the trait (Lettre et al., 2008; Weedon et al., 2008), the effect of an SNP on the trait is estimated with error and this can also cause an SNP that is not the nearest to the QTL to have the largest effect. By using more than 1 independent trait to map the QTL, the second source of error can be reduced but not the first.

Genetic correlations between traits could be attributed to QTL that have pleiotropic effects on multiple traits or could be attributed to closely linked QTL each affecting different traits. Assuming that some QTL show pleiotropy, the pattern of pleiotropic effects would be an important clue to the nature of the causative mutation and the function of the gene in which it occurred. Pleioptropic effects also can be investigated by adjusting one trait with another; this will remove almost all of the effect of that trait.

The objectives of this study were to test 2 simple multivariate methods to detect SNP affecting dairy traits, to understand the patterns of pleiotropic effects of genes that affect an economic index of production traits, and to examine the ability of multiple-trait analysis to increase the precision with which QTL are mapped.

\section{MATERIALS AND METHODS}

\section{Animals and Traits}

The discovery data set, in which the initial GWAS was performed, included 767 Holstein bulls with progeny tests before 2005. The validation data sets were 386 Holstein bulls proven between 2005 and 2007 (validation data set I) and 317 Jersey sires (validation data set II). The bulls had EBV, including information from their daughters, for 9 traits (listed in Table 1). The EBV were extracted from the Australian Dairy Herd Improvement Scheme (ADHIS; Melbourne, Victoria, Australia) database. Three additional traits not routinely evaluated by ADHIS were also included. Persistency of milk yield (persistency) was calculated from the slope of the lactation curve (Pryce et al., 2010b). The sensitivity of milk yield to level of feeding (slope Feed) and heat stress (slope TempHum) were calculated from random regression analyses in which an indicator of these 2 environmental factors was included in the statistical model (Hayes et al., 2009). The Australian selection index (ASI) was also included as a trait. Australian selection index is a selection index comprising milk, fat, and protein yields and reflects the relative value of these traits under a typical Australian milk payment system (ADHIS, 2010). Because a bull's breeding value includes both pedigree information and daughters' phenotypic production (adjusted for fixed effects), significant SNP may arise as a consequence of pedigree information rather than phenotype. Therefore, deregressed EBV were calculated by removing information from relatives (Pryce et al., 2010a). Deregressed EBV have similar properties to daughter yield deviations.

\section{Molecular Markers}

Single nucleotide polymorphism genotypes were obtained from the BovineSNP50 BeadChip (Illumina, San Diego, CA). Preliminary edits were carried out as follows: SNP were discarded if they did not have a call rate greater than $90 \%(41,044 / 56,024 \mathrm{SNP}$ met this criteria), if the proportion of missing genotypes was greater than $20 \%$, if the minor allele frequency was less than 2\% (39,915/41,044 SNP met this criteria), and if SNP departed from Hardy-Weinberg equilibrium at threshold of $P<0.0001$ (636 SNP failed this criteria). The SNP were ordered by chromosome position using Bovine Genome Build 4.0 (http://www.ncbi.nlm. nih.gov/projects/genome/guide/cow/). The SNP that could not be mapped or that were on the X chromosome were excluded, leaving a final set of SNP that was used for the GWAS of 39,048 SNP. Missing genotypes were imputed using the fastPhase program (Scheet and Stephens, 2006). Hayes et al. (2009) reported that the accuracy of imputation of missing genotypes using fastPhase in similar data was $98.7 \%$.

\section{Statistical Analyses}

Single-Trait Analyses. The association between each SNP and each of the traits was assessed by a regression analysis, using ASReml software (Gilmour et al., 2002). The model was as follows:

$$
\mathbf{y}=\mathbf{1}_{n}{ }^{\prime} \mu+\mathbf{w} a+\mathbf{Z u}+\mathbf{e}
$$

where $\mathbf{y}$ is the vector of deregressed $\mathrm{EBV} ; \mathbf{1}_{n}$ is a vector of 1 s of length $n$ sires; $\mu$ is the mean; w is a vector of the SNP genotypes for the SNP being tested $(0,1$, or 
Table 1. Estimated reliability of deregressed EBV for each trait in 3 data sets

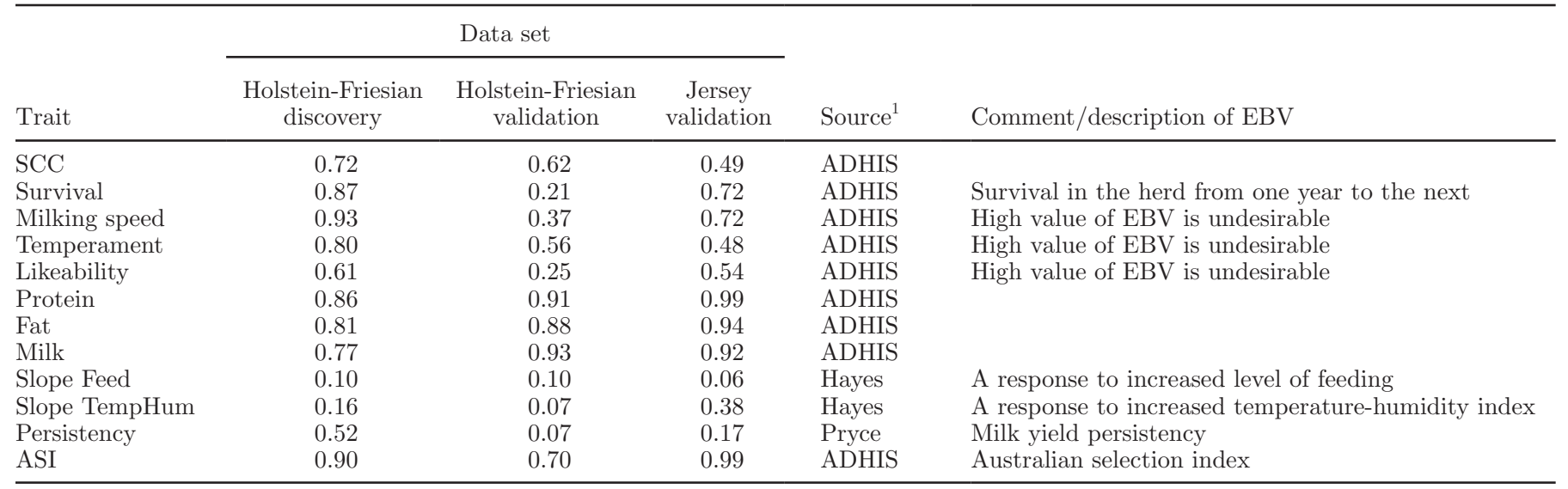

${ }^{1}$ ADHIS = Australian Dairy Herd Improvement Scheme database; Pryce = Pryce et al. (2010b); Hayes = Hayes et al. $(2009)$.

$2) ; a$ is the fixed effect of the SNP; $\mathbf{Z}$ is an incidence matrix relating observations to the corresponding random effect; $\mathbf{u}$ is the vector of polygenic breeding values, sampled from the distribution $\mathrm{N}\left(0, \mathbf{A} \sigma^{2}\right)$, where $\mathbf{A}$ is the numerator relationship matrix; and $\mathbf{e}$ is the vector of random errors. This regression analysis was carried out in the Holstein discovery data set and then the significant SNP were tested in Holstein and Jersey validation data sets. If a polygenic term is not included in the model, many more false positive SNP associations are found. This occurs because related animals tend to share the same SNP genotype and so there is confounding between the polygenic effect and the effect of a single SNP. The polygenic term is not of great importance in itself but is important to minimize false discoveries.

Using the same model without fitting $\operatorname{SNP}_{\mathrm{i}}(\mathbf{w} a)$, estimates of genetic variance of deregressed EBV for each trait in the populations studied were calculated based only on the genotyped animals and their ancestors. The genetic variance as a proportion of the phenotypic variance of deregressed EBV is a measure of the reliability of the EBV. This will be referred to as the estimated reliability to distinguish it from the reliability of the EBV provided by ADHIS.

Four different $P$-values $(0.001,0.01,0.03$, and 0.05$)$ were used. In the discovery data set, 39,048 significance tests were performed for each trait; therefore, a stringent $P$-value $(P<0.001)$ was chosen to reduce false discoveries. In the validation data sets the number of significance tests is much less (hundreds) and, consequently, a $P$-value of 0.01 or 0.05 was used. In the bivariate analysis of the discovery data set, 2 independent tests were used with $P<0.03$ because this is approximately equivalent to 1 test at $P<0.001(0.03$ $\times 0.03 \approx 0.001)$.
Two different types of FDR were used in this study. The traditional FDR was calculated as FDR $=\frac{p(1-s)}{s(1-p)}$, where $p$ is the significance threshold ( $P$-value) and $s$ is a proportion of SNP that are nominally significant (number of significant SNP divided by number of total SNP). This is equivalent to the FDR formula of Storey (2002). This calculation of FDR uses only the discovery data set and relies on the correctness of the $P$-values. A more empirical estimate of FDR can be made using the validation data sets to determine which associations, found in the discovery data set, can be confirmed. The simplest estimate would be based on the proportion of SNP that are significant in the validation data. However, this counts some false positives (i.e., some SNP are significant by chance) but also misses some SNP whose effect is real but too small to be significant in the validation data. An attempt to address the lack of power to detect small but real SNP effects in the validation data was made as follows. Among the SNP (N) that are significant in the discovery data and hence tested in the validation data, assume that those (M) with a real association with the trait will show an effect in the same direction as in the discovery data. Half of the remaining SNP will show an effect in the same direction by chance; consequently, the total number of SNP with effects in the same direction as in the discovery data (Q) is expected to be $\mathrm{M}+(\mathrm{N}-\mathrm{M}) / 2$. Therefore, $\mathrm{M}$ can be estimated by $2 \times \mathrm{Q}-\mathrm{N}$ if $\mathrm{Q} / \mathrm{n} \geq 0.50$ or $\mathrm{M}=0$ if $\mathrm{Q} / \mathrm{n}<0.50$. Using $\mathrm{M}$, another estimate of the FDR can be made. This FDR is referred to as the realized FDR ( $\mathbf{r F D R}$ ) and was calculated as $\mathrm{rFDR}=$ $(\mathrm{N}-\mathrm{M}) / \mathrm{n}=2 \times(\mathrm{N}-\mathrm{Q}) / \mathrm{N}$.

Among the SNP that are significant in both the discovery and validation data sets, some are expected to 
be significant just by chance given the number of SNP tested. This proportion has also been estimated using both FDR and rFDR but this time based only on the subset of SNP that were significant in the discovery data and hence tested in the validation data.

Multiple-Trait Analyses. Analysis of GWAS can be computationally intensive, and the first aim of the study was to test 2 multivariate methods that are simple and fast to apply. When correlated traits are analyzed, the sampling errors tend to be correlated and this makes the interpretation of the results difficult. For instance, it is difficult to distinguish whether the effect of an SNP detected in 2 correlated traits is a result of the presence of a QTL or the same false discovery made twice because of the correlated errors in the 2 traits. To overcome this problem, 2 different methods that generate uncorrelated traits were used: principal components (PC) and bivariate analyses in which one trait is corrected for the other.

The first approach for multivariate analyses using uncorrelated traits was based on PC. Principal component analyses were performed on the trait-by-trait correlation matrix to convert the set of (correlated) traits to a smaller number of linear combinations of uncorrelated variables called PC. The PC were computed based on the correlation matrix of deregressed EBV in a discovery data set using the statistical package GenStat (Payne et al., 2007). The 11 traits in Table 1 (not including ASI) were used to compute $\mathrm{PC}$. The $\mathrm{PC}$ are linear combinations of the 11 traits: $\mathbf{u}_{k}=\mathbf{T}^{\prime} \mathbf{g}_{k}$, where $\mathbf{u}_{k}$ is an $11 \times 1$ vector of PC scores for animal $k, \mathbf{g}_{k}$ is an $11 \times 1$ vector of deregressed and standardized EBV for animal $k$, and $\mathbf{T}$ is an $11 \times 11$ matrix of eigenvectors such that the variance matrix of the $\mathrm{PC} \operatorname{Var}\left(\mathbf{T}^{\prime} \mathbf{g}\right)=\mathbf{D}$, a diagonal matrix of eigenvalues. Although $11 \mathrm{PC}$ are generated, only the first 5 that explain $71.3 \%$ of total variance were retained for further analysis.

The scores of each of the $5 \mathrm{PC}$ were used as phenotypes and analyzed using the same linear model as the single traits. An F-statistic for the effect of each SNP on each $\mathrm{PC}$ was calculated. An approximate $F$-test with 5 numerator degrees of freedom was calculated by averaging the $5 F$-values for each SNP from the $5 \mathrm{PC}$ analyzed (PCt5). This tests the null hypothesis that the SNP has no effect on any of the PC. To the extent that the first $5 \mathrm{PC}$ capture all the meaningful variance of the 11 traits, this also tests the null hypothesis that the SNP has no effect on any of the 11 traits. The purpose of the PC analysis was to have a simple method of testing this multiple-trait null hypothesis; the PC themselves are not of interest.

The SNP that were significant in the multiple-trait $F$-test were tested on PC scores in the validated data sets. The PC scores in the validation data sets were obtained using the same transformation as in the discovery data set (i.e., using the eigenvectors calculated in the discovery data set). The confirmation of an association in the validation populations can be made more powerful by testing for an effect along the same multidimensional axis as found in the discovery population. This was done by computing a linear combination of the PC scores (s) that maximizes the effect of the SNP in the discovery population: $\mathbf{s}=\mathbf{b}^{\prime} \mathbf{u}$, where $\mathbf{b}$ is a vector with element $\mathbf{b}_{i}=$ effect of the SNP on the $i$ th $\mathrm{PC}$, and $\mathbf{u}$ is the same vector of PC score as defined above. The corresponding SNP in the validation data set was tested for its effect on this new trait (s). A second PC analysis was carried out that used only the 3 milk production traits (milk, fat, and protein yields) and retained all $3 \mathrm{PC}$.

The second bivariate method considered pairs of traits that consisted of ASI and 1 other trait. The second trait was corrected for ASI using the regression of the trait on ASI. That is, an ASI-corrected trait $\left(\mathrm{y}_{\mathrm{c}}\right)$ was calculated as $\mathrm{y}_{\mathrm{c}}=\mathrm{y}-\beta(\mathrm{ASI})$, where $\beta$ is the regression of the trait $\mathrm{y}$ on a vector of deregressed EBV of ASI. This created a pair of uncorrelated traits, and then the probability that the SNP has an effect on one trait is independent of the effect on the other trait. Single SNP analyses were performed for the traits. The aim here was to find SNP that had a significant effect on both traits. This procedure can be used in 2 ways: 1) to find SNP for ASI based on first screening all SNP for an effect on another trait that has been corrected for ASI and then testing for their effect on ASI, or 2) to find SNP for the second trait based on first screening all SNP for an effect on ASI and then testing the significant SNP for significance on the other trait. The SNP associations discovered in this way in the discovery population were then tested in the validation populations.

Pleiotropy. The SNP that are associated with ASI are likely to be associated with other milk production traits. For each SNP with a significant effect on ASI, its effects were examined on 4 other traits corrected for ASI (protein yield, fat yield, milk volume, and persistency) and on protein percentage and fat percentage.

Precision of Mapping a $Q T L$. The ability of multiple-trait analysis to increase the precision with which QTL are mapped was examined. The genotyped SNP are not expected to be the cause of an effect on the trait but merely in LD with the causal polymorphism or QTL. It is useful to know how far from the causal QTL the significant SNP is likely to be. Because the position of the QTL is not known, the distance is not directly observable. Instead, the distance between the significant SNP in the discovery and validation populations was calculated. The SNP significant at $P<0.001$ were identified in the discovery data set. If there was 
Table 2. Correlations between deregressed EBV in the Holstein discovery data set ${ }^{1}$

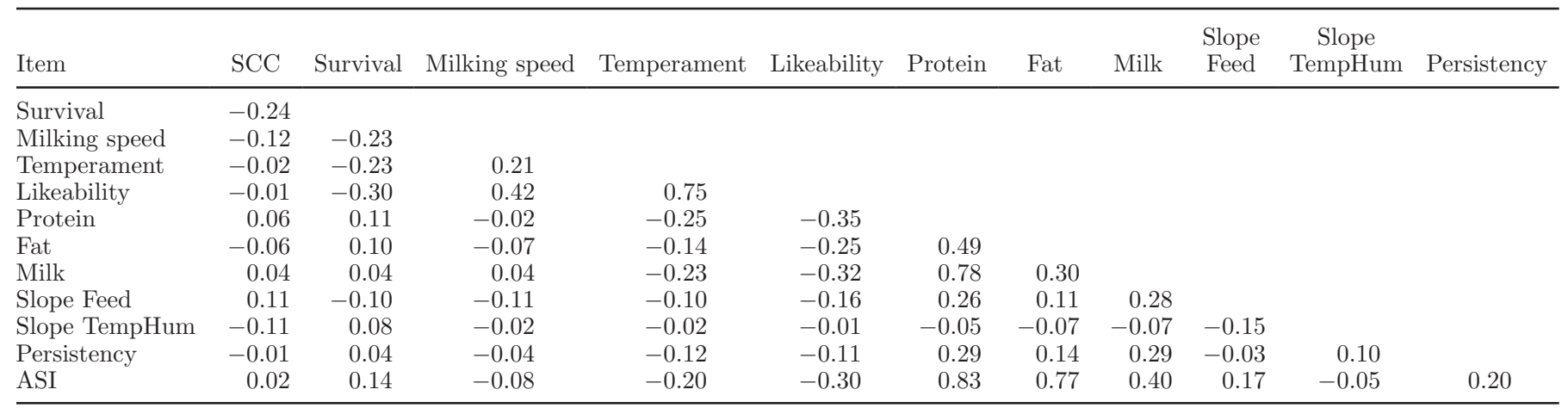

${ }^{1}$ Slope Feed $=$ a response to increased level of feeding; Slope TempHum = a response to increased temperature-humidity index; ASI = Australian selection index.

more than 1 significant SNP within a specified length of interval (in the discovery population), then only the most significant SNP was retained because it is likely that they are all tracking the same QTL. For each SNP retained from the discovery population, the SNP 0 to $0.5 \mathrm{Mbp}, 0.5$ to $1.0 \mathrm{Mbp}$, and up to 4.5 to $5.0 \mathrm{Mbp}$ either side of this position were examined in the validation populations. The number of significant $(P<0.01)$ SNP in the validation data set were counted in these intervals. Each interval is $1 \mathrm{Mbp}$ in size because it consists of $0.5 \mathrm{Mbp}$ on either side of the discovery SNP. The number of expected and observed SNP within 1-Mbp intervals was compared using a chi-squared test. The expected number of significant SNP within any 1-Mbp interval in the validation data $\left(\mathrm{P}_{\mathrm{e}}\right)$ is the total number of significant SNP divided by the total genome length of 2,544 Mbp. Therefore, the expected number $\left(\mathrm{N}_{\mathrm{e}}\right)$ within $\mathrm{N}_{\mathrm{o}} 1-\mathrm{Mbp}$ intervals is $\mathrm{N}_{\mathrm{e}}=\mathrm{N}_{\mathrm{o}} \times \mathrm{P}_{\mathrm{e}}$, where $\mathrm{N}_{\mathrm{o}}$ is the number of SNP retained in the discovery data.

\section{RESULTS}

\section{PC Analysis}

For the traits evaluated by ADHIS, the reliability of EBV for the bulls in the discovery data set was greater than 0.94 except SCC, for which the reliability was 0.88 (P. Bowman, Department of Primary Industries, Bundoora, Victoria, Australia; personal communication). The estimated reliability of the deregressed EBV used for the 12 traits is shown in Table 1. The estimated reliabilities were high for production traits but low for sensitivity of milk yield to level of feeding and heat stress. Out of the 12 traits, the 3 production traits (protein yield, fat yield, and milk yield) were highly and positively correlated with each other (Table 2). Workability traits were less correlated, but in a favorable direction, with survival and production traits. Eleven traits (not including ASI) were used in the PC analysis and the first 5 PC, which explain $71.3 \%$ of total variance, were retained (Table 3 ).

\section{Genome-Wide Association Results}

Single Traits. An analysis of some of these traits has already been reported by Pryce et al. (2010a), but the number of significant SNP and associated FDR are included here for completeness. Table 4 gives the results of testing 39,048 SNP for association with each trait and with the PC. For protein yield, 169 SNP (169/39,048 $=0.0043)$ were significant $(P<0.001)$ in the discovery population, many more than the 39 significant SNP expected by chance. Consequently, the FDR is $23 \%$ $[=0.001 \times 0.996 /(0.004 \times 0.999)]$. When these 169 SNP were tested in the Holstein validation population, $78.1 \%$ (132 SNP) showed an effect on protein yield in the same direction as the discovery population, which is an $\mathrm{rFDR}$ of $44 \%$ [=2 $\times(169-132) / 169]$. Alternately, the number of true associations (M) is $2 \times 132-169$ $=95$. The difference between these 2 estimates of FDR (23 vs. $44 \%$ ) could indicate that the significance test in the discovery population is not conservative enough or that the validation population, given its small size, lacks power to find an effect in the same direction even though the association is real.

When these 169 SNP were tested in the Holstein validation data set, 60 were significant $(P<0.05)$. This is less than the number of real associations calculated (95) because demanding that the effect is significant is a more restrictive criterion than merely that it is in the same direction and, consequently, more true associations fail to achieve significance in the validation data set. However, even some of these 60 significant associations are expected to be false discoveries. When 169 significance tests are made at a $P$-value of $0.05,169$ $\times 0.05=8$ significant SNP were expected by chance. In fact, 60 out of $169(36 \%)$ were significant $(P<0.05)$ in the validation population for protein, which is an FDR 
Table 3. Summary of the principal components (PC) in the Holstein discovery data set

\begin{tabular}{|c|c|c|c|c|c|c|c|c|}
\hline Item $^{1}$ & \multicolumn{5}{|c|}{ First 5 PC calculated from 11 traits } & \multicolumn{3}{|c|}{ PC calculated from 3 traits } \\
\hline Eigenvalue & 2.84 & 1.70 & 1.41 & 1.05 & 0.85 & 2.08 & 0.74 & 0.19 \\
\hline \multicolumn{9}{|l|}{ Direction of vectors } \\
\hline $\mathrm{SCC}$ & 0.03 & -0.27 & -0.39 & -0.60 & 0.28 & & & \\
\hline Survival & 0.18 & 0.46 & 0.09 & 0.37 & 0.03 & & & \\
\hline Likeability & -0.47 & -0.29 & 0.20 & 0.08 & -0.06 & & & \\
\hline Slope Feed & 0.23 & -0.34 & -0.25 & 0.06 & -0.57 & & & \\
\hline Slope TempHum & -0.06 & 0.33 & 0.26 & -0.41 & -0.69 & & & \\
\hline Persistency & -0.10 & 0.27 & 0.42 & -0.45 & 0.25 & & & \\
\hline Protein & 0.46 & -0.24 & 0.32 & -0.09 & 0.01 & -0.46 & 0.86 & 0.21 \\
\hline Fat & 0.32 & -0.12 & 0.24 & 0.26 & 0.16 & -0.60 & -0.48 & 0.64 \\
\hline Milk & 0.42 & -0.28 & 0.32 & -0.14 & -0.05 & -0.64 & -0.17 & -0.74 \\
\hline
\end{tabular}

${ }^{1}$ Slope Feed $=$ a response to increased level of feeding; Slope TempHum $=$ a response to increased temperature-humidity index; no. of SNP $=$ number of significant SNP in the discovery data set.

among these 60 of $9.6 \%$. Also, an rFDR can be estimated as $17 \%$ among these 60 significant SNP based on the fact that, within these 60 SNP, $91.7 \%$ had an effect in the same direction in both data sets (Table 4).

Of the 12 traits, those with low reliabilities of their EBV had the highest FDR, the lowest number of SNP confirmed in the validation sample, and a high proportion of false discoveries even among these confirmed SNP. When the significant SNP for the 3 production traits were combined, there were 433 unique SNP significant $(P<0.001)$ in the discovery population (Table 4).

$\boldsymbol{P C}$. When the effect of the SNP was tested across the $5 \mathrm{PC}$ derived from all 11 traits, there were 139, 81, 69, 121, and 62 SNP significant for the first 5 PC. When the $5 \mathrm{PC}$ were combined and a single significance test was performed (PC5t), 204 SNP were significant in the discovery population $(\mathrm{FDR}=19.1 \%)$. This and the other statistics for PCt5 are similar to those for the production traits despite the fact that these $\mathrm{PC}$ contain information from the other 8 traits, which show much higher FDR. When only the 3 production traits are used to calculate PC (PCt3), 514 SNP are significant in the combined $F$-test in the discovery population $(\mathrm{FDR}=7.5 \%$; Table 4$)$. This is confirmed by the high proportion (86.1\%) of 514 SNP with an effect in the same direction in the Holstein validation population. In fact, 193 of the 514 SNP were significant $(P<0.05)$ in the Holstein validation population. Further, $97.9 \%$ of these 193 SNP have an effect in the same direction in the discovery and validation populations (Table 4), indicating that most of these 193 associations are not attributable to chance.

The results of testing the significant SNP in the Jersey population are shown in Table 4 . The number of
SNP tested is slightly less than in the Holstein validation population because some SNP were monomorphic in Jerseys. The proportion of these SNP with an effect in the same direction in Jerseys as in Holsteins was approximately $50 \%$ for all traits. This is not surprising because the phase of LD is likely to be different in Jerseys than in Holsteins (de Roos et al., 2008) at the marker spacing used in this study. Therefore, it is not expected that the same SNP allele will consistently be associated with increased ASI in both Jerseys and Holsteins. However, very few of the SNP significant in Holsteins were also significant in Jerseys. For most traits the number was no more than expected by chance. For production traits, slightly more SNP were significant than was expected by chance. In particular, for the combined effect of the SNP on the 3 PC calculated from milk, fat, and protein, 95 out of 491 SNP were significant in Jerseys compared with 193 out of 514 in Holsteins. For ASI, more SNP were significant in the Jersey validation set (40) than in the Holstein validation set (27). However, this is the only case where this occurred and is probably the result of sampling variation.

Bivariate Analyses. The rationale for the bivariate analyses is that an SNP is unlikely to be significantly associated with 2 uncorrelated traits by chance alone. However, if an SNP is in LD with a QTL that affects both traits (because of pleiotropy), the SNP will show an association with both traits. Therefore, SNP that have a significant association with 2 uncorrelated traits are more likely to have a true association with a QTL than SNP discovered by a single-trait analysis. Tables 5 and 6 show the result of testing this hypothesis. The probability of finding the same SNP significant when tested for its effect on each trait independently at $P$ $<0.03$ for 2 independent traits is $P<0.0009 \approx 0.001$. 
Table 4. Number and false discovery rate of SNP in the discovery data set $(P<0.001)$ and proportion of the SNP effects with same direction in the Holstein and Jersey validation data sets ${ }^{1}$

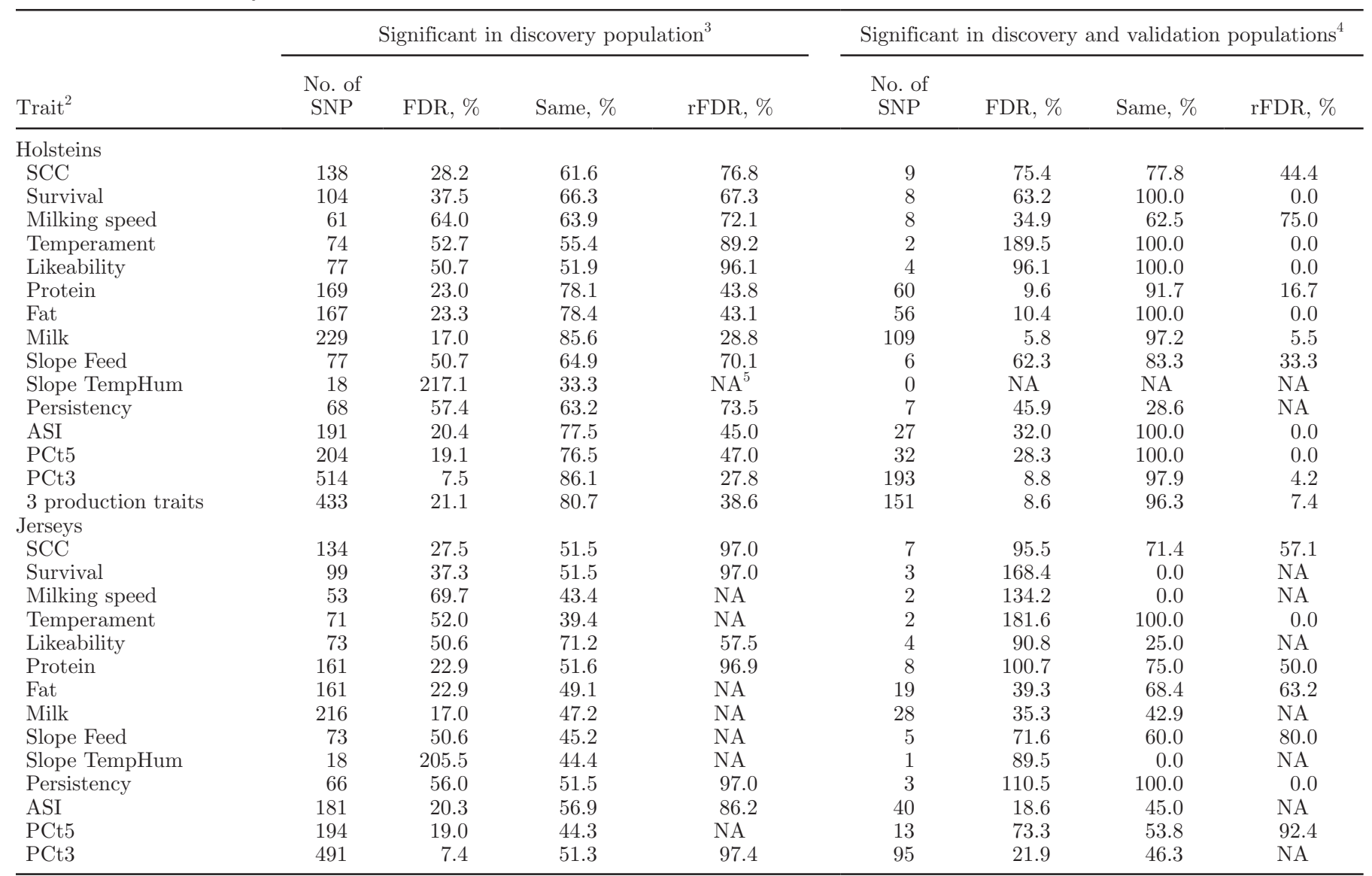

${ }^{1}$ No. of SNP = number of SNP selected at different thresholds; FDR = false discovery rate; same = SNP having same direction in the validation data set as in the discovery data set; rFDR $=$ released false discovery rate.

${ }^{2}$ Slope Feed $=$ a response to increased level of feeding; Slope TempHum = a response to increased temperature-humidity index; ASI $=$ Australian selection index; PCt5 = first 5 principal components $(\mathrm{PC})$ calculated from 11 traits; PCt3 = PC calculated from 3 traits.

${ }^{3}$ Single nucleotide polymorphisms significant $(P<0.001)$ for the specified trait in the discovery data set.

${ }^{4}$ Significant SNP for the specified trait $(P<0.001)$ in the discovery data set were significant $(P<0.05)$ in the validation data set.

${ }^{5} \mathrm{NA}=$ not applicable.

For example, 2,026 SNP were significant $(P<0.03)$ for protein corrected for ASI in the discovery population and, of these, 212 were also significant $(P<0.03)$ for ASI. Because $2,026 \times 0.03=61$ would be expected by chance, the FDR among these $212 \mathrm{SNP}$ is estimated to be $27 \%$ :

$$
\begin{aligned}
& {\left[\operatorname{FDR}(\%)=\frac{p(1-s)}{s(1-p)} \times 100 \%\right.} \\
& \left.=\frac{0.03(1-212 / 2,026)}{(212 / 2,026)(1-0.03)} \times 100 \% \approx 27 \%\right] .
\end{aligned}
$$

This FDR is worse than the $20 \%$ reported in Table 4 when ASI alone was examined using a significance level of 0.001 . Of these 212 SNP, $81.6 \%$ had an effect in the same direction for ASI as the Holstein validation population, which implies an rFDR of $37 \%$. In fact, 27 of these SNP were significantly associated with ASI $(P$ $<0.05)$ in the Holstein validation population and 42 in the Jersey validation population compared with 27 and 40, respectively, for the 191 SNP selected only on ASI (Table 4). Similar results were obtained for milk and fat but for other traits the bivariate strategy failed to find as many validated SNP for ASI as the single-trait analysis. This may be because relatively few SNP had significant effects on both ASI and another trait. For instance, at $P<0.03,2,277 \mathrm{SNP}$ were significant for ASI and 2,194 for SCC corrected for ASI, and 101 for both. Because 39,048 SNP were tested, it is expected that $2,194 \times 2,277 / 39,048=128$ would be significant for both even if there was no tendency for SNP to affect 
Table 5. Single nucleotide polymorphisms significant for ASI after testing only those SNP significant for each trait corrected for Australian selection index $(\mathrm{ASI})^{1}$

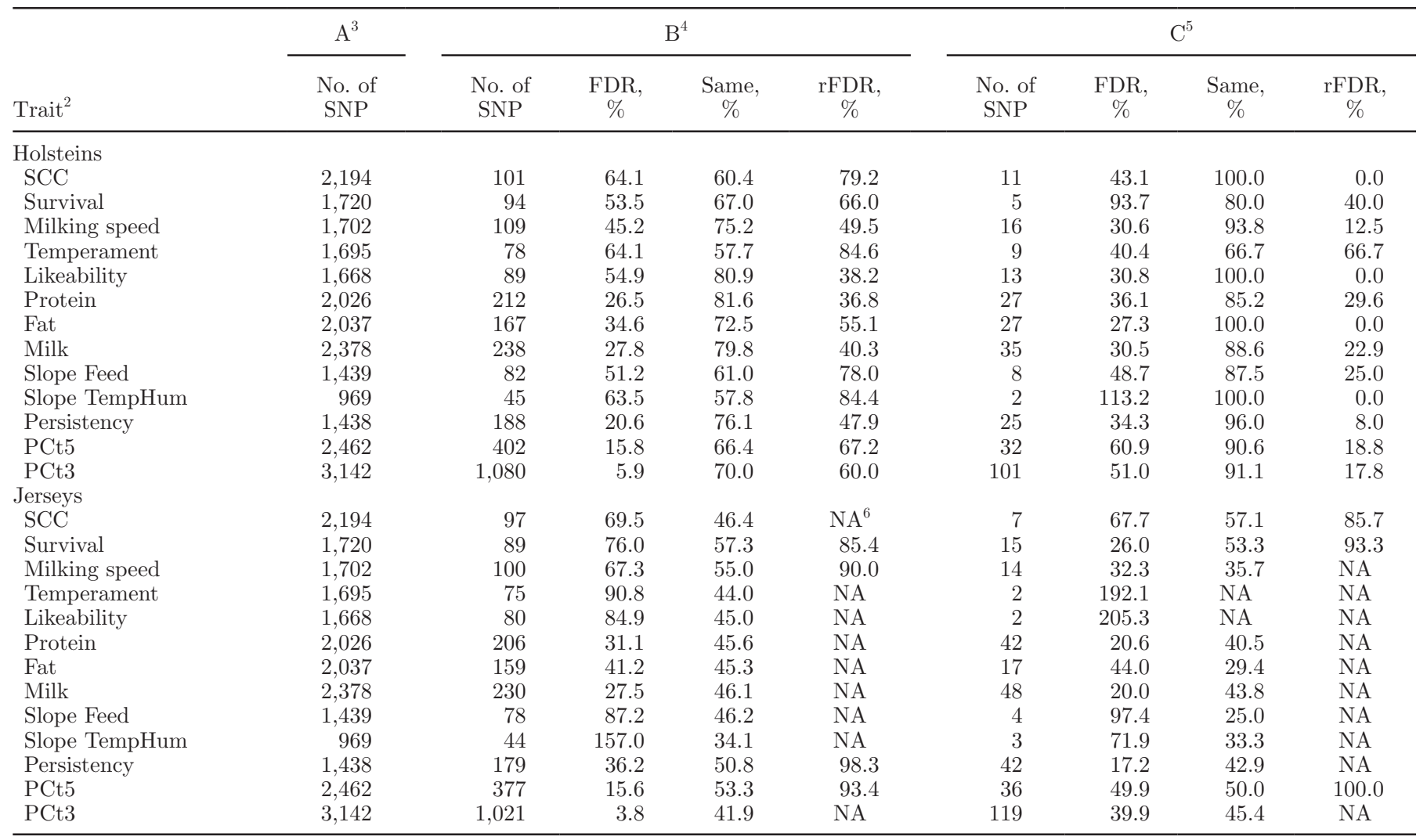

${ }^{1}$ No. of SNP = number of SNP selected at different thresholds; FDR = false discovery rate; same = SNP having same direction in the validation data set as in the discovery data set; $r F D R=$ released false discovery rate.

${ }^{2}$ Slope Feed $=$ a response to increased level of feeding; Slope TempHum $=$ a response to increased temperature-humidity index; PCt5 $=$ first 5 principal components $(\mathrm{PC})$ calculated from 11 traits; $\mathrm{PCt} 3=\mathrm{PC}$ calculated from 3 traits.

${ }^{3}$ Single nucleotide polymorphisms significant $(P<0.03)$ for the specified trait in the discovery data set.

${ }^{4}$ Significant SNP $(P<0.03)$ for ASI in the discovery data set that were significant $(P<0.03)$ for the specified trait in the discovery data set.

${ }^{5}$ Single nucleotide polymorphisms in B that were significant $(P<0.05)$ for ASI alone in the validation data set.

${ }^{6} \mathrm{NA}=$ not applicable.

both traits. However, the low power to detect SNP for many of the nonproduction traits make them less useful for identifying SNP with an effect on ASI.

Table 5 shows the results of selecting SNP that were significant for ASI and in 1 of the 2 PC analyses. Because the PC are not independent from ASI, it is not surprising that many SNP were significant for the PC and for ASI. However, of the 1,080 SNP discovered using the 3 trait PC, 101 were significant for ASI in the Holstein validation population and 119 out of 1,021 tested were significant in the Jersey population. Of the 101 significant in both Holstein populations, $91.1 \%$ have an effect in the same direction, giving rFDR of $17.8 \%$. In Jerseys the proportion of SNP with an effect in the same direction is approximately 50\%, again presumably because the linkage phase between the SNP and the causal mutation is not preserved from Holstein to
Jersey even though the causal mutation may segregate in both breeds.

Among the 212 SNP that were significant for ASI and for protein corrected for ASI in the discovery population, 27 had a significant association with ASI in the Holstein validation population. By coincidence, 27 SNP were also significantly associated with ASI in the Holstein validation population out of the 191 significant for ASI $(P<0.001)$ in the discovery population. However, these 2 sets of $27 \mathrm{SNP}$ were not the same. For instance, of the 212 SNP, 67 were also included in the 191 that were SNP significant for ASI only and the remaining $145(=212-67)$ were not among these 191 SNP (Table 7). Of the 145, 17 were significant in the Holstein validation population and these were new confirmed SNP not included in the 27 significant in the Holstein validation population after discovery on ASI 
Table 6. Single nucleotide polymorphisms significant for each trait not corrected for Australian selection index (ASI) after testing only those SNP significant for $\mathrm{ASI}^{1}$

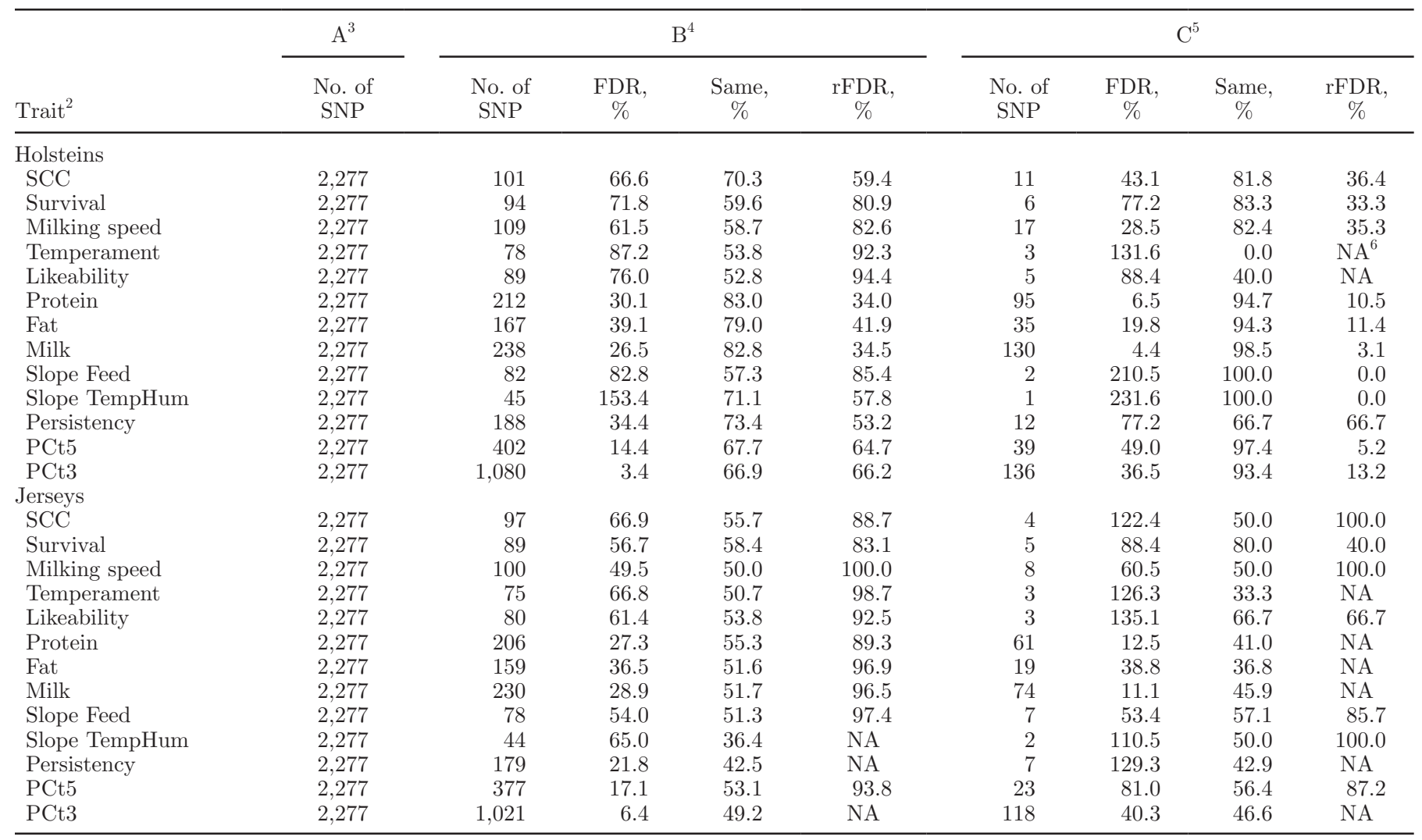

${ }^{1}$ No. of SNP = number of SNP selected at different thresholds; FDR $=$ false discovery rate; same = SNP having same direction in the validation data set as in the discovery data set; $\mathrm{rFDR}=$ released false discovery rate.

${ }^{2}$ Slope Feed $=$ a response to increased level of feeding; Slope TempHum $=$ a response to increased temperature-humidity index; PCt $5=$ first 5 principal components $(\mathrm{PC})$ calculated from 11 traits; PCt3 = PC calculated from 3 traits.

${ }^{3}$ Single nucleotide polymorphisms significant $(P<0.03)$ for ASI in the discovery data set.

${ }^{4}$ Significant SNP $(P<0.03)$ for the specified trait in the discovery data set that were significant $(P<0.03)$ for ASI in the discovery data set.

${ }^{5}$ Single nucleotide polymorphisms in B that were significant $(P<0.05)$ for the specified trait alone in the validation data set.

${ }^{6} \mathrm{NA}=$ not applicable.

alone (Table 7). Therefore, the bivariate approach did add to the number of SNP discovered for ASI without greatly increasing the FDR.

Table 6 shows the results of the bivariate strategy for identifying SNP that were associated with other traits based on first screening for a significant effect on ASI. For instance, out of the 212 SNP that were significant for the bivariate analysis of ASI and protein corrected for ASI in the discovery population, 95 were significant for protein corrected for ASI in the Holstein validation and 61 in the Jerseys. This can be compared with the 60 and $8 \mathrm{SNP}$ in the Holstein and Jersey validation populations, respectively, when SNP were discovered based on protein alone (Table 4). Table 8 shows that of the $95 \mathrm{SNP}$ discovered and confirmed in Holsteins for protein corrected for ASI, 84 were not discovered using protein alone. However, although these 84 SNP were significant for protein corrected for ASI, only 3 were significant for protein itself in the Holstein validation population. This reflects the fact that protein and ASI are highly correlated; therefore, protein corrected for ASI is a very different trait than protein. Because ASI comprises milk, fat, and protein, protein corrected for ASI could be linked to either fat synthesis or milk volume.

This marked difference is not apparent for all traits. For instance, the bivariate analysis discovered and confirmed 10 SNP for SCC (Table 8) that were not among the 9 discovered and confirmed using SCC only (Table $4)$.

\section{Patterns of Pleiotropy}

There were 829 SNP in the HF discovery, 473 in the $\mathrm{HF}$ validation, and 472 in the Jersey data sets, which were significant at $P<0.03$ for ASI as well as for 1 or 
Table 7. Number of extra SNP revealed by screening Australian selection index (ASI) on corrected trait

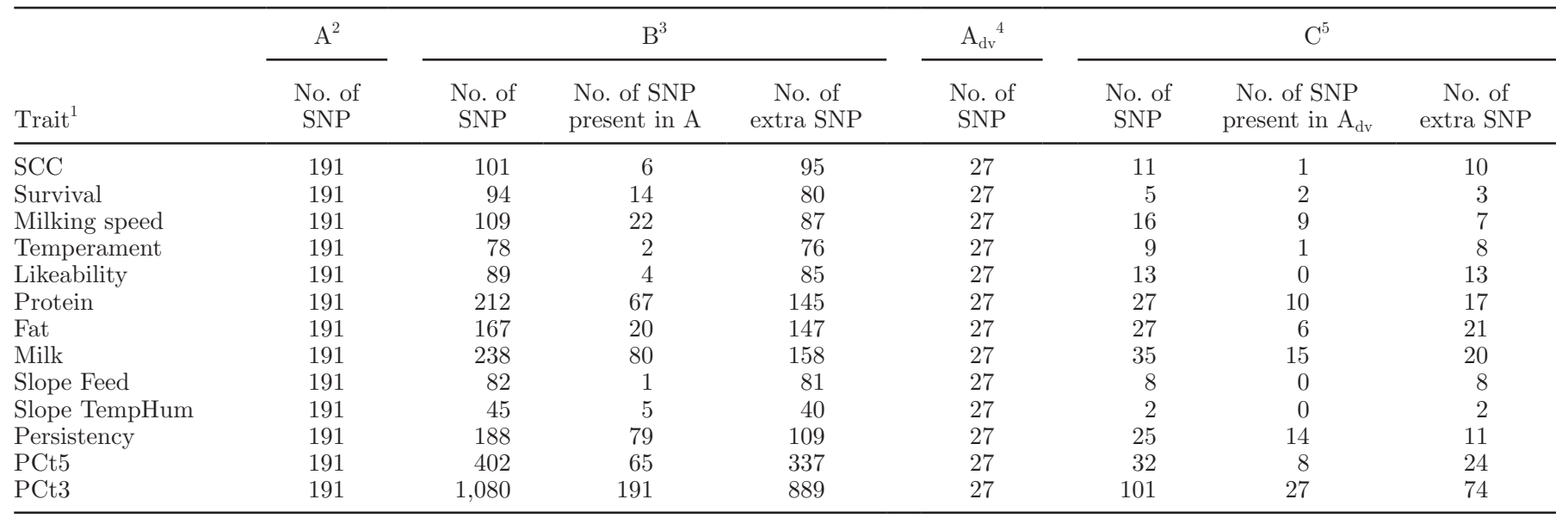

${ }^{1}$ Slope Feed $=$ a response to increased level of feeding; Slope TempHum $=$ a response to increased temperature-humidity index; PCt5 $=$ first 5 principal components $(\mathrm{PC})$ calculated from 11 traits; $\mathrm{PCt} 3=\mathrm{PC}$ calculated from 3 traits.

${ }^{2}$ Single nucleotide polymorphisms significant $(P<0.001)$ for ASI in the discovery data set.

${ }^{3}$ Significant SNP $(P<0.03)$ for ASI in the discovery data set that were significant $(P<0.03)$ for the corrected trait in the discovery data set.

${ }^{4}$ Significant SNP for ASI $(P<0.001)$ in the discovery data set that were significant $(P<0.05)$ in the validation data set.

${ }^{5}$ Single nucleotide polymorphisms in B that were also significant $(P<0.05)$ for the corrected trait alone in the validation data set.

more of the 6 traits (4 traits corrected for ASI and uncorrected protein percentage and fat percentage), giving a total of $1,774(=829+473+472)$ different SNP $\times$ data set combinations (cases). These 1,774 cases were grouped into 27 different pleiotropic patterns based on the direction of the effect of the SNP on each of the 4 traits (Table 9). Because the choice of a base allele is arbitrary, it has been chosen for each SNP so that the effect on ASI is positive. Table 9 shows that the most common pattern, accounting for 504 of the 1,774 cases, is one in which the SNP allele that increases ASI decreases protein, fat, milk, and persistency corrected for ASI but increases protein and fat percentages. The next most common 3 patterns are similar to the first except that the effect on fat or persistency or both is positive but seldom significant. Thus, in the 4 most

Table 8. Number of extra SNP revealed by screening uncorrected trait on Australian selection index (ASI)

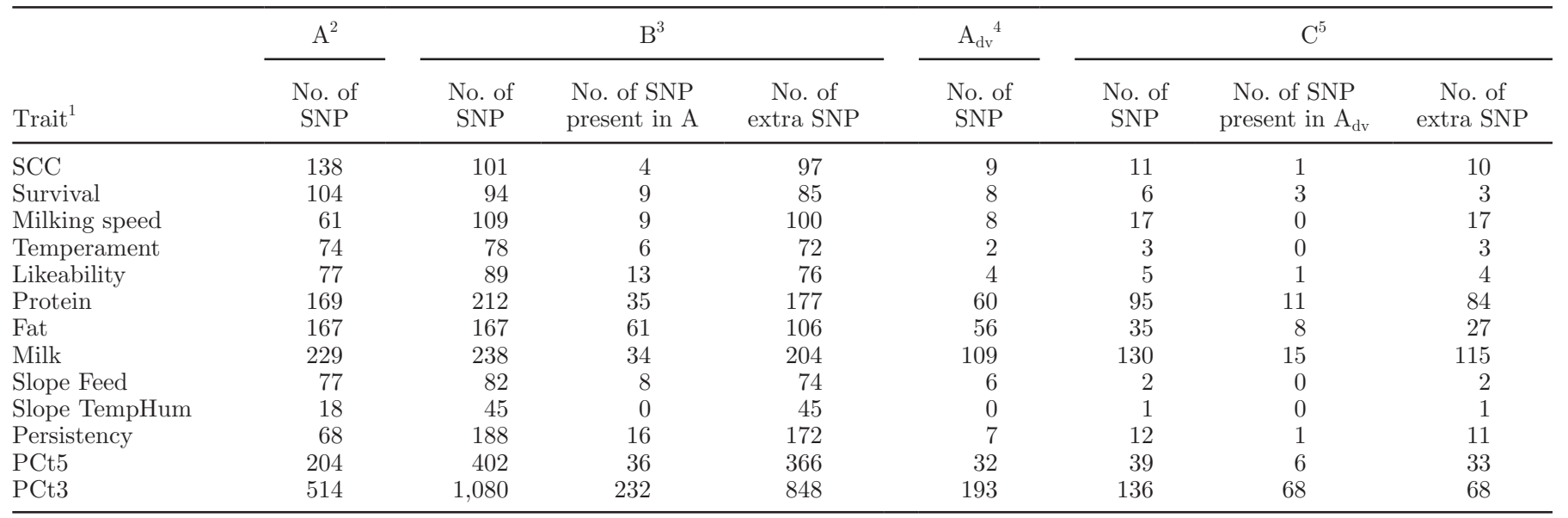

${ }^{1}$ Slope Feed $=$ a response to increased level of feeding; Slope TempHum $=$ a response to increased temperature-humidity index; PCt $5=$ first 5 principal components $(\mathrm{PC})$ calculated from 11 traits; PCt3 = PC calculated from 3 traits.

${ }^{2}$ Single nucleotide polymorphisms significant $(P<0.001)$ for the uncorrected trait in the discovery data set.

${ }^{3}$ Significant SNP $(P<0.03)$ for the corrected trait in the discovery data set that were significant $(P<0.03)$ for ASI in the discovery data set. ${ }^{4}$ Significant SNP for the uncorrected trait $(P<0.001)$ in the discovery data set that were significant $(P<0.05)$ in the validation data set.

${ }^{5}$ Single nucleotide polymorphisms in B that were also significant $(P<0.05)$ for the uncorrected trait alone in the validation data set. 
Table 9. Pleiotropic patterns affecting Australian selection index (ASI), fat percentage, protein percentage, and other 4 traits corrected for ASI

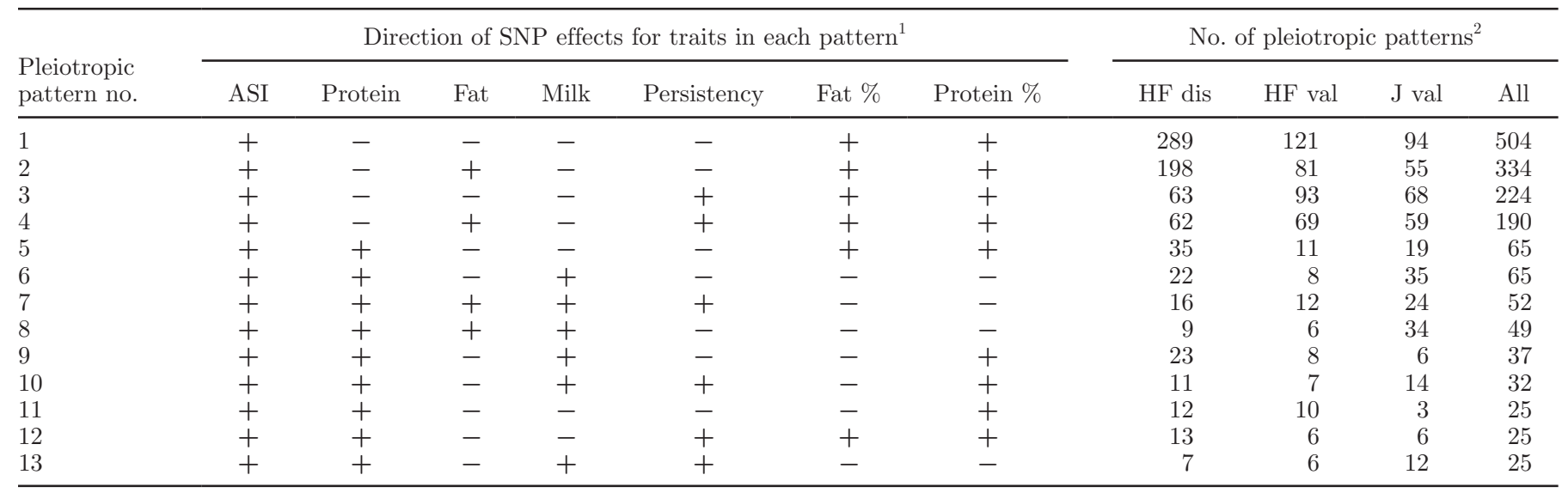

${ }^{1}$ Single nucleotide polymorphisms significant for ASI $(P<0.03)$ are significant $(P<0.03)$ for 1 or more of the 6 traits $(4$ traits corrected for ASI and uncorrected protein percentage and fat percentage) and the direction of SNP effects for the traits that are included in each global pleiotropic pattern denoted as + or - [compared with the direction of ASI QTL effect $(+)$, SNP effects having same $(+)$ or opposite $(-)$ directions].

${ }^{2} \mathrm{HF}=$ Holstein-Friesian; $\mathrm{J}=$ Jersey; dis = discovery data set; val = validation data set.

common pleiotropic patterns, the allele that increases ASI decreases milk and protein corrected for ASI and increases fat and protein percentages.

To help interpret these pleiotropic patterns, Table 10 divides the cases in the first 4 patterns according to their effect on uncorrected milk, fat, and protein. In each case there are 2 subpatterns or types: one in which the allele that increases ASI also increases milk, fat, and protein and one in which it increases fat and protein but decreases milk. The subpattern in which milk volume is decreased is the more common and the decrease is often significant, whereas the increase in milk yield is only significant in 1 case. Thus, these 4 patterns are characterized by increasing protein and fat with constant or decreasing milk so that fat and protein percentages increase. Because ASI has a negative weighting for milk, this causes ASI to increase more than fat or protein and therefore fat and protein corrected for ASI decline. The 4 patterns differ mainly in the extent to which fat increases: in patterns 2 and 4 fat increases faster than expected for the effect on ASI and may in some cases be the main contributor to the increase in ASI.

Patterns 5, 11, and 12 are similar to patterns 1 through 4 except that protein corrected for ASI increases. In this case, the increase in ASI is largely driven by an increase in protein yield with little or no increase in milk and hence an increase in protein percentage.

Patterns 6 through 10 and 13 show increases in milk and protein corrected for ASI and decreases in percentages of fat and protein. These are SNP where the allele that increases ASI increases milk more than protein and especially more than fat. These patterns were more common in Jerseys than in Holsteins.

\section{Precision of Mapping QTL}

The potential advantage of multiple-trait analyses to position the QTL more precisely than single-trait analyses was also investigated. For each trait, the number of significant SNP in the validation population clustered around the position of a significant SNP in the discovery population was examined. First, the set of significant $(P<0.001)$ SNP in the discovery population was reduced by retaining only the most significant SNP within any 1-Mbp interval. For instance, this reduced the number of significant SNP from 138 to 99 for SCC (Table 11). Then, the significant $(P<$ $0.01) \mathrm{SNP}$ in the validation population were counted within $0.5 \mathrm{Mbp}$ of each significant discovery SNP (e.g., 28 for SCC in Table 11). Table 11 shows the number of significant SNP within each of ten 1-Mbp intervals surrounding each discovery SNP. For most traits this number declines as the distance from the discovery SNP is increased. For instance, for fat yield there are 67 validation SNP within $0.5 \mathrm{Mbp}$ of a discovery SNP, 51 within 0.5 to $1 \mathrm{Mbp}, 47$ within 1 to $1.5 \mathrm{Mbp}$, and so on (Table 11). Only for traits in which the ability to validate SNP was very weak, such as sensitivity of milk yield to level of heat stress, was there a tendency for the number of significant SNP to decrease as the distance from the position of the discovery SNP increased. The number of significant validation SNP per megabase can be compared with the number expected by chance. For instance, for SCC there were 549 significant SNP in the validation population in a total genome length of 2,544 Mbp; therefore, 549/2,544/Mbp was expected. The total number of SNP in ninety-nine 1-Mbp intervals was counted. Therefore, $99 \times 549 / 2,544=21$ were expected 


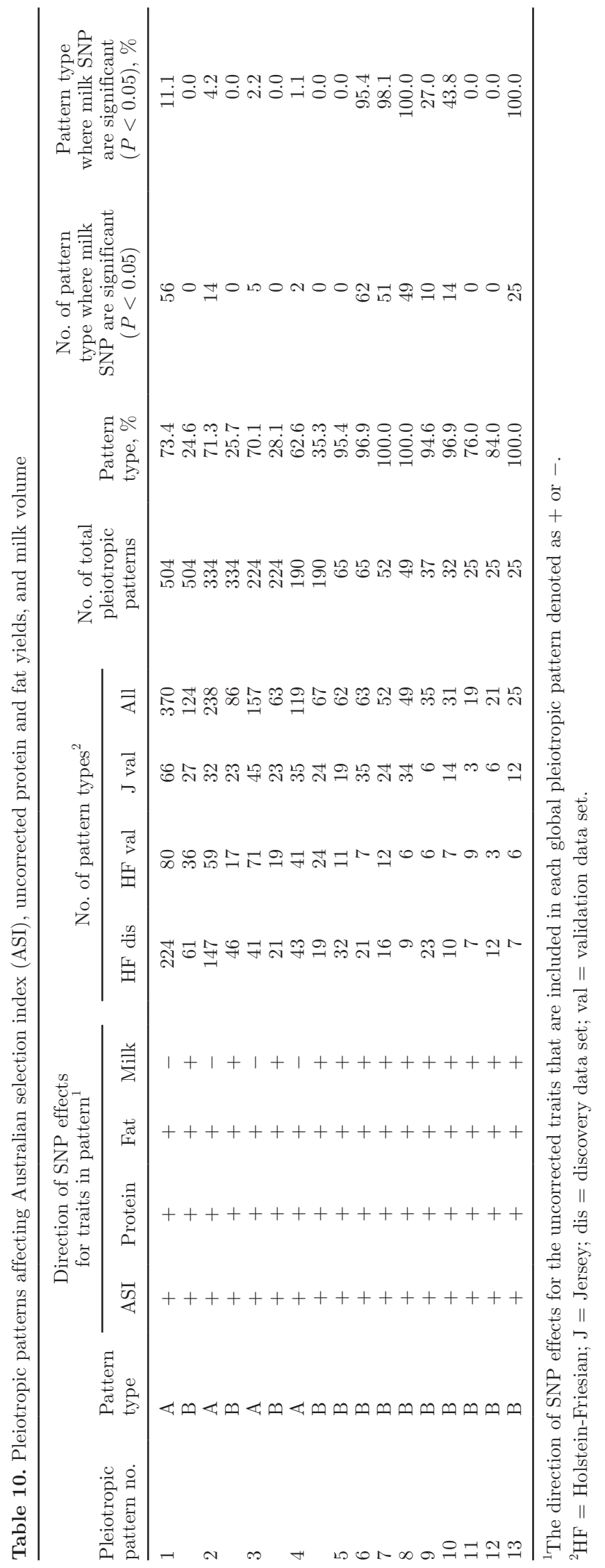

within a 1-Mbp interval containing the discovery SNP. As Table 11 shows, the number within $0.5 \mathrm{Mbp}$ of the discovery SNP is usually greater than expected by chance, but the number in the interval 4.5 to $5.0 \mathrm{Mbp}$ from a discovery SNP is often no more than expected by chance.

Table 11 also shows the PC analyses of 11 traits and of 3 traits. The results are similar to those observed for traits such as milk volume, fat, and protein yields, which show the greatest clustering of significant validation SNP around discovery SNP (chi-squared, $P<$ $0.001)$.

\section{DISCUSSION}

The results from the single-trait analyses showed that for traits in which the phenotype (i.e., the deregressed EBV) had a low relative proportion of genetic variance relative to phenotypic variance (e.g., reliability of the deregressed EBV), few significant SNP were detected, in some cases no more than expected by chance. This is because either the true heritability was low (e.g., survival) or there were few daughters contributing to the EBV. For the traits that relied on a random regression on production level or temperature or stage of lactation, there is less genetic variance and consequently the EBV have low reliability. In particular, sensitivity to heat stress shows little variation under Australian conditions and therefore it is not surprising that no convincing SNP associations were found. By contrast, the milk production trait EBV had high reliability and many confirmed associations were detected with SNP.

In the Holstein validation population, the proportion of SNP that had an effect in the same direction as in the Holstein discovery population was more than 0.5 for most traits. In the Jersey validation population, this proportion was around 0.5 for all traits. This does not necessarily indicate that the QTL, discovered in Holsteins, do not segregate in Jerseys, but could indicate that the phase of LD between an SNP and a QTL is not preserved between Jerseys and Holsteins (de Roos et al., 2008). For most traits, the number of SNP discovered in Holsteins that were significant in Jerseys was less than the number significant in the Holstein validation population. This may indicate that these QTL explain less variance in Jerseys than Holsteins because of a lower minor allele frequency.

In GWAS it is often useful to have a validation population that is not closely related to the discovery population, and in this study the Jersey population was a useful validation population. Validation within Holsteins might be criticized because the Holsteins in the validation population are related (e.g., offspring or grand offspring) to the Holsteins in the discovery population. 
This criticism cannot be made of the Jersey validation population. The 3 trait $\mathrm{PC}$ analysis confirmed that 95 SNP discovered in Holsteins as significant in Jerseys. This indicates that at least some of the QTL segregating in Holsteins are also segregating in Jerseys.

Two estimates of the FDR (FDR and rFDR) were compared. In most cases the rFDR was lower than the FDR, perhaps because the effects of some SNP are very small and sampling error can cause the estimated effects to be in opposite directions in the discovery and validation data sets. The rFDR was not useful in the Jersey validation data because, as pointed out previously, LD phase is not the same in Holsteins and Jerseys.

Principal component and other multivariate analyses have been used to detect QTL with linkage mapping (Chase et al., 2002; Stearns et al., 2005; Musani et al., 2006). These methods have been reported to increase power. Here, a PC analysis on the (co)variance matrix among the traits was performed, and then the GWAS was conducted on the PC. This study shows that the power of the multiple-trait analysis conducted in this way was approximately as high as that of the singletrait analyses for the production traits. However, a PC analysis that used only the 3 production traits confirmed more SNP in the Jersey validation than for any single trait (Table 4).

One interpretation might be that the inclusion of traits with low reliability in the PC analysis decreased the power. Principal components were used in this study to identify SNP that had an effect on multivariate phenotypes (PCt5 and PCt3). Therefore, QTL for the first 5 or $3 \mathrm{PC}$ are not discussed individually; instead, the total PC (PC5t or PCt3) were investigated and compared with the results from single-trait analysis. Further, Stearns et al. (2005) pointed out that the relative advantage of multivariate over the univariate approaches varied with the level of genetic covariance between traits. Among the 11 traits that were analyzed, there are many low correlations and this may have also reduced the benefits from a multivariate approach.

It is normal practice in $\mathrm{PC}$ analysis to retain only the $\mathrm{PC}$ with eigenvalue $>1$. One additional $\mathrm{PC}$ was retained to capture more of the total variance. The decision to keep only 5 PC from the 11 trait analysis was based on the assumption that the remaining variance would be largely noise because the remaining $\mathrm{PC}$ had small eigenvalues $(<0.8)$. However, it appears that applying this decision means that some of the genetic variance of important traits is lost. For instance, only 11 of the 191 significant SNP for ASI were also significant at $P$ $<0.001$ for PCt5. By contrast, the PC analysis of only the 3 production traits, where all $3 \mathrm{PC}$ were retained, found 116 of these 191 SNP significant at $P<0.001$.
Also, a bivariate approach in which ASI and 1 other trait were analyzed together was investigated. One purpose of these analyses was to find SNP significantly associated with ASI that were not significant when ASI was analyzed alone. Generally, more significant SNP were not identified using a bivariate analysis for ASI than when the univariate analysis was used. However, the significant SNP found using the bivariate analysis had an FDR similar to the univariate analysis (Table 5) and there were often nonsignificant SNP in the univariate analysis (Table 7). Alternatively, the bivariate analysis could be considered as a method for finding SNP associated with a second trait by first screening all the SNP for those that were significant for ASI. This was most beneficial for traits with low reliable EBV in which the univariate analysis found few confirmed associations. For instance, the univariate analysis of SCC found only $7 \mathrm{SNP}$ confirmed in the Holstein validation population (Table 4) but the bivariate method found 11, 10 of which had not been found in the univariate analysis (Table 7).

Australian selection index is an economic index of milk production traits. Selection is often practiced on an index of several traits and therefore the following question arises: "Should SNP associated with the index be discovered directly by an analysis of the index, or should they be discovered by analysis of the contributing traits?" The results here suggest the use of both approaches if there is a subsequent validation of the SNP discovered. In fact, when the PC analysis of the 3 traits was used as a screen to identify SNP associated with ASI, 74 additional SNP were identified for ASI that were confirmed in the Holstein validation population (Table 7).

For SNP associated with ASI, the pattern of pleiotropic effects was investigated on other traits. There are 3 main patterns of pleiotropic effects. The first and most common pattern is an increase in protein yield combined with a decrease or no change in milk yield so that protein percentage increases. The SNP showing this pattern probably can be divided into 2 groups: those in which the increase in ASI is mainly attributed to increasing protein and those in which it is mainly attributed to decreasing milk volume. The third group of SNP achieves an increase in ASI by increasing protein but not as much as milk so that protein percentage declines. This type of SNP is more common in Jerseys than in Holsteins. There is a fourth group of SNP in which the increase in ASI is mainly driven by an increase in fat yield and, hence, fat percentage increases. These 4 groups roughly correspond to the 4 types of QTL found using linkage mapping by Chamberlain et al. (2005). These authors interpreted them as genes that 
Table 11. Number of SNP in the Holstein-Friesian discovery data set (HF dis; $P<0.001)$ and the HF validation data set (HF val; $P<0.01$ ), the expected and observed number of SNP in each 1-Mbp interval, and number of significant SNP in 10 different intervals in the HF validation data set

\begin{tabular}{|c|c|c|c|c|c|c|c|c|c|c|c|c|c|c|c|c|}
\hline \multirow[b]{2}{*}{ Item $^{1}$} & \multicolumn{3}{|c|}{ No. of $\mathrm{SNP}^{2}$} & \multirow[b]{2}{*}{ Overlaps $^{3}$} & \multirow[b]{2}{*}{$\begin{array}{c}\mathrm{N}_{\mathrm{e}} \text { of SNP } \\
\text { in 1-Mbp } \\
\text { interval }^{4}\end{array}$} & \multicolumn{11}{|c|}{ No. of significant $\mathrm{SNP}^{5}$} \\
\hline & $\begin{array}{c}\text { HF dis } \\
(P< \\
0.001)\end{array}$ & $\begin{array}{c}\text { HF val } \\
(P<0.01)\end{array}$ & $\begin{array}{c}\text { Reduced HF } \\
\quad \text { dis } \\
(P<0.001)\end{array}$ & & & $\begin{array}{l}0-0.5 \\
\text { Mbp }\end{array}$ & $\begin{array}{l}0.5-1 \\
\text { Mbp }\end{array}$ & $\begin{array}{l}1-1.5 \\
\text { Mbp }\end{array}$ & $\begin{array}{l}1.5-2 \\
\text { Mbp }\end{array}$ & $\begin{array}{l}2-2.5 \\
\text { Mbp }\end{array}$ & $\begin{array}{l}2.5-3 \\
\text { Mbp }\end{array}$ & $\begin{array}{l}3-3.5 \\
\text { Mbp }\end{array}$ & $\begin{array}{l}3.5-4 \\
\text { Mbp }\end{array}$ & $\begin{array}{l}4-4.5 \\
\text { Mbp }\end{array}$ & $\begin{array}{l}4.5-5 \\
\text { Mbp }\end{array}$ & Total \\
\hline SCC & 138 & 549 & 99 & 1 & 21 & 28 & 18 & 21 & 19 & 20 & 21 & 33 & 17 & 19 & 16 & 212 \\
\hline Survival & 104 & 396 & 72 & 2 & 11 & 13 & 9 & 7 & 8 & 10 & 10 & 10 & 12 & 5 & 5 & 89 \\
\hline Milking speed & 61 & 496 & 47 & 2 & 9 & $21^{* * *}$ & 20 & 25 & 15 & 20 & 11 & 18 & 21 & 15 & 23 & 189 \\
\hline Temperament & 74 & 598 & 58 & 0 & 14 & 9 & 7 & 9 & 11 & 16 & 12 & 10 & 4 & 12 & 13 & 103 \\
\hline Likeability & 77 & 564 & 63 & 0 & 14 & 13 & 18 & 12 & 13 & 6 & 16 & 14 & 19 & 20 & 15 & 146 \\
\hline Protein & 169 & 573 & 73 & 5 & 16 & $46^{* * *}$ & 41 & 31 & 47 & 34 & 37 & 37 & 26 & 29 & 30 & 358 \\
\hline Fat & 167 & 576 & 90 & 8 & 20 & $67^{* * *}$ & 51 & 47 & 45 & 36 & 35 & 29 & 30 & 23 & 24 & 387 \\
\hline Milk & 229 & 729 & 113 & 17 & 32 & $159^{* * *}$ & 104 & 117 & 111 & 82 & 106 & 95 & 102 & 75 & 108 & 1,059 \\
\hline Slope Feed & 77 & 434 & 53 & 0 & 9 & 12 & 10 & 11 & 7 & 9 & 5 & 9 & 5 & 8 & 9 & 85 \\
\hline Slope TempHum & 18 & 323 & 16 & 0 & 2 & 2 & 2 & 0 & 4 & 2 & 3 & 4 & 4 & 5 & 1 & 27 \\
\hline Persistency & 68 & 486 & 48 & 0 & 9 & 11 & 11 & 10 & 10 & 7 & 3 & 6 & 6 & 9 & 3 & 76 \\
\hline ASI & 191 & 618 & 106 & 3 & 26 & $42^{* *}$ & 25 & 25 & 32 & 20 & 23 & 26 & 27 & 33 & 22 & 275 \\
\hline PCt5 & 204 & 588 & 115 & 3 & 27 & 30 & 29 & 17 & 27 & 18 & 21 & 23 & 18 & 16 & 37 & 236 \\
\hline PCt3 & 514 & 717 & 221 & 32 & 153 & $252^{* * *}$ & 213 & 192 & 188 & 167 & 172 & 177 & 177 & 149 & 163 & 1,850 \\
\hline
\end{tabular}

${ }^{1}$ Slope Feed $=$ a response to increased level of feeding; Slope TempHum = a response to increased temperature-humidity index; ASI $=$ Australian selection index; PCt $5=$ first 5 principal components $(\mathrm{PC})$ calculated from 11 traits; PCt3 $=$ PC calculated from 3 traits.

${ }^{2}$ No. of SNP, HF dis $(P<0.001)=$ number of significant SNP at $P<0.001$ in the HF dis population; no. of SNP. HF val $(P<0.01)=$ number of significant SNP at $P<0.01$ in the HF val population; no. of SNP, reduced HF dis $(P<0.001)=$ number of significant SNP at $P<0.001$ in the HF discovery population excluding the 1 -Mbp intervals, which do not include any significant SNP.

을 $\quad{ }^{3}$ Overlaps $=$ significant $\mathrm{SNP}$ at same position in HF dis (reduced) and HF val data sets.

ב ${ }^{4}$ Expected number of SNP within one 1-Mbp interval $[0.5 \mathrm{Mbp}$ on either side of each of significant SNP $(P<0.001)$ in discovery data set].

을 ${ }^{5}$ The SNP that were significant in the discovery population were examined in the validation population by counting all SNP that were significant on either side of the SNP tested. The intervals tested were $0-0.5 \mathrm{Mbp}, 0.5-1.0$, and up to $4.5-5.0$ 
increased synthesis of protein, genes that decrease synthesis of lactose, genes that increase milk production in general, and genes that increase fat synthesis. However, the results in this study seem less clear cut, perhaps because genes that primarily affect synthesis of one milk component also affect synthesis of other components possibly through competition for substrate. The known acyl-CoA:diacylglycerol acyltransferase gene (DGAT1; Grisart et al., 2002; Spelman et al., 2002) is an example of these secondary effects; DGAT1 primarily affects fat synthesis, but the DGAT1 genotypes also differ in milk and protein yield.

The group of genes that increase milk yield more than protein and fat yields have effects that resemble those of some nongenetic factors such as stage of lactation and age and may reflect a physiological pathway that stimulates lactation but to which lactose synthesis is more sensitive than fat and protein synthesis. Lillehammer et al. (2008) also found genes that alter a cow's milk yield in response to feeding level but have less effect on fat and protein. Our results suggest that these genes have a bigger role to play in increasing ASI in Jerseys than in Holsteins. A possible mechanism by which these genes might act is suggested by the results of Chagas et al. (2009). They found that Holsteins of North American origin had higher milk yields, but not protein and fat yields, than Holsteins of New Zealand origin, possibly in part because of increased resistance to insulin.

It is possible that the association between an SNP and more than 1 trait reflects the effect of multiple QTL each affecting a single trait rather than 1 QTL affecting multiple traits. With the current density of SNP marker panels it is difficult to distinguish multiple QTL in close proximity. It may be possible to distinguish between these QTL using denser SNP panels.

The precision of mapping was investigated by counting significant SNP in the validation data set on either side of every significant SNP location in the discovery data set in ten $1-\mathrm{Mbp}$ windows (range: $0.5-5 \mathrm{Mbp}$ at either side of significant SNP). The number of significant SNP was greatest within $0.5 \mathrm{Mbp}$ of the discovery SNP but the number remained above that expected by chance even in the 4.5 to $5.0-\mathrm{Mbp}$ interval for traits such as milk yield. MacLeod et al. (2009), in a simulation study based on Holstein SNP data, also found that the most significant SNP could be $5 \mathrm{Mbp}$ from the QTL because of the long-range LD in Holsteins and the environmental noise associated with estimating the small SNP effects. The PC analyses give a similar clustering of confirmed SNP in the $<0.5-\mathrm{Mbp}$ window as the 3 milk production traits.
The SNP may show an association with a trait because of LD between the SNP and a causal polymorphism. The LD may extend for $<10 \mathrm{Mbp}$ in cattle (de Roos et al., 2008). If significant SNP are consistently found in a validation population very close to the position of the significant SNP in the discovery population, then the causal polymorphism is probably mapped relatively precisely. This study presents evidence that significant SNP are found more often than expected by chance within $0.5 \mathrm{Mbp}$ of the significant SNP in the discovery population.

Visscher et al. (1996) applied the bootstrap method to determine an empirical confidence interval of a QTL location found by a linkage analysis. The authors found strong negative correlations between the test statistic and the width of the confidence interval, hence the stronger the evidence of a QTL segregating the smaller the empirical confidence interval for its location. The results in this study show a similar trend, with the greatest clustering of confirmed SNP for milk yield that had the most significant SNP.

\section{CONCLUSIONS}

The power of single-trait GWAS to detect SNP associated with a trait depends on the correlation between the phenotype used and the breeding value for that trait (i.e., the reliability of the EBV where that is the phenotype used in the GWAS). This study identified that the power of a multiple-trait GWAS was at least as high as that of single-trait GWAS for the traits with the highest reliability of EBV. Also, the benefit of a multiple-trait analysis over a single-trait analysis increased if the traits were highly correlated. Consequently, the $\mathrm{PC}$ analysis based on 3 traits found more confirmed SNP associations than the PC analysis based on 11 traits because the 3 traits used were those that were most highly correlated and with the most reliable EBV. The multivariate analyses, both PC and bivariate, did discover additional confirmed SNP associations that were not discovered by the univariate analyses without increasing the FDR. The SNP associated with effects on ASI tend to show 1 of 4 pleiotropic patterns. These patterns could correspond to QTL whose primary effects are to increase protein synthesis, decrease lactose synthesis, increase fat synthesis, or stimulate lactation in general, but lactose synthesis more than fat and protein synthesis. However, these patterns are not clear-cut, possibly because the primary effect is accompanied by secondary effects on other milk components due to competition for substrate or other causes. In the validation population, significant SNP tended to cluster 
within $0.5 \mathrm{Mbp}$ of an SNP that was significant in the discovery population, but there was still an excess of significant SNP up to $5 \mathrm{Mbp}$ away from the discovery SNP. Principal component analysis did not noticeably improve the precision of mapping QTL.

\section{ACKNOWLEDGMENTS}

The authors thank ADHIS (Melbourne, Victoria, Australia) for providing the phenotype data used in this study and Phil Bowman (Department of Primary Industries, Bundoora, Victoria, Australia) for extracting the dataset from the ADHIS database and calculating the deregressed EBV.

\section{REFERENCES}

ADHIS. 2010. Australian Dairy Herd Improvement Scheme. www. adhis.org.au Accessed Nov. 1, 2009.

Benjamini, Y., and Y. Hochberg. 1995. Controlling the false discovery rate: A practical and powerful approach to multiple testing. J. R. Stat. Soc., B 57:289-300.

Chagas, L. M., M. C. Lucy, P. J. Back, D. Blache, J. M. Lee, P. J. Gore, A. J. Sheahan, and J. R. Roche. 2009. Insulin resistance in divergent strains of Holstein-Friesian dairy cows offered fresh pasture and increasing amounts of concentrate in early lactation. J. Dairy Sci. 92:216-222.

Chamberlain, A. J., T. H. E. Meuwissen, and M. E. Goddard. 2005. Estimation of the distribution of QTL effects. Proc. Assoc. Advmt. Anim. Breed. Genet. 16:103-106.

Chase, K., D. R. Carrier, F. R. Adler, T. Jarvik, E. A. Ostrander, and T. D. Lorentzen. 2002. Genetic basis for systems of skeletal quantitative traits: Principal component analysis of the canid skeleton. Proc. Natl. Acad. Sci. USA 99:9930-9935.

Cole, J. B., P. M. VanRaden, J. R. O'Connell, C. P. Van Tassell, T. S. Sonstegard, R. D. Schnabel, J. F. Taylor, and G. R. Wiggans. 2009. Distribution and location of genetic effects for dairy traits . J. Dairy Sci. 92:2931-2946.

de Roos, A. P., B. Hayes, R. Spelman, and M. Goddard. 2008. Linkage disequilibrium and persistence of phase in Holstein-Friesian, Jersey and Angus cattle. Genetics 179:1503-1512.

Gilmour, A. R., B. J. Gogel, B. R. Gullis, S. J. Welham, and R. Thompson. 2002. ASReml User Guide. Release 1.0. VSN International Ltd., Hemel Hempstead, UK.

Grisart, B., W. Coppitiers, F. Farnir, L. Karim, C. Ford, P. Berzi, N. Cambisano, M. Mni, S. Reid, P. Simon, R. Spelman, M. Georges, and R. Snell. 2002. Positional candidate cloning of a QTL in dairy cattle: Identification of a missense mutation in the bovine DGAT1 gene with major effect on milk yield and composition. Genome Res. 12:222-231.

Hayes, B., P. Bowman, A. Chamberlain, and M. Goddard. 2009. A validated genome-wide association study to breed cattle adapted to an environment altered by climate change. PLoS One 4:e6676.

Karlsson, E. K., I. Baranowska, C. M. Wade, N. H. C. S. Hillbertz, M. C. Zody, N. Anderson, T. M. Biagi, N. Patterson, G. R. Pielberg, E. J. Kulbokas, K. E. Comstock, E. T. Keller, J. P. Mesirov, H. von Euler, O. Kaumlmpe, A. Hedhammar, E. S. Lander, G. Andersson, L. Andersson, and K. Lindblad-Toh. 2007. Efficient mapping of mendelian traits in dogs through genome-wide association. Nat. Genet. 39:1321-1328.

Knott, S. A., and C. S. Haley. 2000. Multitrait least squares for quantitative trait loci detection. Genetics 156:899-911.
Korol, A. B., Y. I. Ronin, A. M. Itskovich, J. Peng, and E. Nevo. 2001. Enhanced efficiency of quantitative trait loci mapping analysis based on multivariate complexes of quantitative traits. Genetics 157:1789-1803.

Lettre, G., A. Jackson, C. Gieger, F. Schumacher, S. Berndt, S. Sanna, S. Eyheramendy, B. Voight, J. Butler, C. Guiducci, T. Illig, R. Hackett, I. Heid, K. Jacobs, V. Lyssenko, and M. Uda. 2008. Identification of ten loci associated with height highlights new biological pathways in human growth. Nat. Genet. 40:584-591.

Lillehammer, M., M. Goddard, H. Nilsen, E. Sehested, G. Olsen, S. Lien, and T. Meuwissen. 2008. QTL by environment interaction for milk yield traits on Bos Taurus Autosome 6. Genetics doi:0.1534/ genetics.1107.084483

MacLeod, I. M., B. J. Hayes, K. W. Savin, A. J. Chamberlain, H. C. McPartlan, and M. E. Goddard. 2009. Power of a genome scan to detect and locate quantitative trait loci in cattle using dense single nucleotide polymorphisms. J. Anim. Breed. Genet. 127:133-142.

Matukumalli, L. K., C. Lawley, R. Schnabel, J. Taylor, M. Allan, M. Heaton, J. O'Connell, S. Moore, T. Smith, T. Sonstegard, and C. Van Tassell. 2009. Development and characterization of a high density SNP genotyping assay for cattle. PLoS ONE 4:e5350.

Musani, S. K., H. Zhang, H. Hsu, N. Yi, B. S. Gorman, D. B. Allison, and J. D. Mountz. 2006. Principal component analysis of quantitative trait loci for immune response to adenovirus in mice. Hereditas 143:189-197.

Osborne, J. 2006. Estimating the false discovery rate using SAS. 19030 in SAS Conference Proceedings: SAS Users Group International 31. http://www2.sas.com/proceedings/sugi31/190-31.pdf Accessed Aug. 10, 2009

Payne, R. W., D. A. Murray, S. A. Harding, D. B. Baird, and D. M. Soutar. 2007. GenStat for Windows. 10th ed. VSN International, Hemel Hempstead, UK.

Pryce, J., M. Haile-Mariam, K. Verbyla, P. Bowman, M. Goddard, and B. Hayes. 2010b. Genetic markers for lactation persistency in primiparous Australian dairy cows. J. Dairy Sci. 93:2202-2214.

Pryce, J. E., S. Bolormaa, A. Chamberlain, P. Bowman, K. Savin, M. Goddard, and B. Hayes. 2010a. A validated genome-wide association study in two dairy cattle breeds for milk production and fertility traits using variable length haplotypes. J. Dairy Sci. 93:3331-3345.

Scheet, P., and M. Stephens. 2006. A fast and flexible statistical model for large-scale population genotype data: Applications to inferring missing genotypes and haplotypic phase. Am. J. Hum. Genet. 78:629-644.

Spelman, R. J., C. Ford, P. McElhinney, G. Gregory, and R. Snell. 2002. Characterization of the DGAT1 gene in the New Zealand dairy population. J. Dairy Sci. 85:3514-3517.

Stearns, T. M., J. E. Beever, B. R. Southey, M. Ellis, F. K. McKeith, and S. L. Rodriguaz-Zas. 2005. Evaluation of approaches to detect quantitative traits loci for growth, carcass, and meat quality on swine chromosome 2, 6, 13 and 18. II. Multivariate and principal component analyses. J. Anim. Sci. 83:2471-2481.

Storey, J. D. 2002. A direct approach to false discovery rates. J. R. Stat. Soc. B 64:479-498.

The Bovine HapMap Consortium. 2009. Genome-wide survey of SNP variation uncovers the genetic structure of cattle breeds. Science 324:528-532.

Visscher, P. M., R. Thompson, and C. S. Haley. 1996. Confidence interval in QTL mapping by bootstrapping. Genetics 143:10131020.

Weedon, M. N., H. Lango, C. Lindgren, C. Wallace, D. Evans, M. Mangino, R. Freathy, J. Perry, S. Stevens, A. Hall, N. Samani, B. Shields, I. Prokopenko, M. Farrall, and A. Dominiczak. 2008. Genome-wide association analysis identifies 20 loci that influence adult height. Nat. Genet. 40:575-583. 